\title{
Mid-term results of laparoscopic Roux-en-Y gastric bypass and laparoscopic sleeve gastrectomy compared-results of the SLEEVEPASS and SM-BOSS trials
}

\author{
David Benaiges $^{1,2,3}$, Elisenda Climent ${ }^{1,2}$, Albert Goday ${ }^{1,2,3}$, Helena Julià ${ }^{1,2}$, Juana A. Flores-Le Roux ${ }^{1,2,3}$, \\ Juan Pedro-Botet ${ }^{1,2,3}$ \\ ${ }^{1}$ Department of Endocrinology and Nutrition, Hospital del Mar, Paseo Marítimo, Barcelona, Spain; ${ }^{2}$ Department of Medicine, Universitat Autònoma \\ de Barcelona, Campus Universitari Mar, Barcelona, Spain; ${ }^{3}$ Institut Hospital del Mar d'Investigacions Mèdiques (IMIM), Barcelona, Spain \\ Correspondence to: Dr. David Benaiges. Department of Endocrinology, Hospital del Mar, Paseo Marítimo, 25-29, E-08003 Barcelona, Spain. \\ Email: 96002@parcdesalutmar.cat. \\ Provenance: This is an invited Editorial commissioned by the Section Editor Kaiping Zhang (AME College, AME Group, China). \\ Comment on: Salminen P, Helmiö M, Ovaska J, et al. Effect of Laparoscopic Sleeve Gastrectomy vs Laparoscopic Roux-en-Y Gastric Bypass on \\ Weight Loss at 5 Years Among Patients With Morbid Obesity: The SLEEVEPASS Randomized Clinical Trial. JAMA 2018;319:241-54. \\ Peterli R, Wölnerhanssen BK, Peters T, et al. Effect of Laparoscopic Sleeve Gastrectomy vs. Laparoscopic Roux-en-Y Gastric Bypass on Weight \\ Loss in Patients With Morbid Obesity: The SM-BOSS Randomized Clinical Trial. JAMA 2018;319:255-65.
}

Submitted Oct 25, 2018. Accepted for publication Oct 29, 2018.

doi: $10.21037 /$ atm.2018.10.70

View this article at: http://dx.doi.org/10.21037/atm.2018.10.70

Two important studies in the field of bariatric surgery have been published recently in a special issue of $\mathcal{F A M A}$ : the Finnish Sleeve vs. Bypass (SLEEVEPASS) and the Swiss Multicenter Bypass or Sleeve Study (SM-BOSS) trials $(1,2)$. These two randomised controlled trials compared the 5-year efficacy of gastric bypass (GB) and sleeve gastrectomy (SG) in patients with severe obesity, and provided significant and novel information, with direct implications for the clinical management of candidates for bariatric surgery.

\section{What did we know before the SLEEVEPASS and SM-BOSS trials?}

The prevalence of obesity has risen worldwide in recent decades, becoming a serious public health issue (3). Patients with an increased body mass index present different comorbidities, including type 2 diabetes mellitus, hypertension and dyslipidaemia, which are partially responsible for the high cardiovascular risk in this population (4).

Management of obesity with lifestyle modifications and/or with pharmacological treatment yields limited results, particularly in terms of long-term weight loss $(5,6)$. On the other hand, bariatric surgery can achieve durable weight loss with resolution of comorbidities and lower mortality (7). Nevertheless, results with different bariatric surgery procedures can be clearly diverse. In this respect, GB has been considered the gold standard in bariatric surgery owing to its better risk/benefit balance compared with restrictive techniques such as lap band or purely malabsorptive techniques such as biliopancreatic diversion (8). GB is a hybrid bariatric surgery technique that combines a restrictive mechanism (gastric resection) with a malabsorptive mechanism (duodenal and proximal jejunum bypass). Its metabolic benefits are mediated by weight loss and various gastro-intestinal-mediated mechanisms (9). For these reasons, GB was the most used bariatric surgery procedure worldwide in the first decade of the 21 th century (10).

SG is a bariatric technique consisting of subtotal vertical gastrectomy with preservation of the pylorus, and includes longitudinal resection of the fundus, corpus and antrum to create a tubular duct along the lesser curvature. This relatively new technique was initially used in a two-step approach to treat patients with high surgical risk (11). Despite being a restrictive technique, SG has yielded similar results to GB in terms of short-term weight loss or type 2 diabetes remission (12). These benefits have been associated 
with different pathophysiological effects unrelated to weight loss such as increased gastric emptying and intestinal transit, and activation of hormonal mechanisms (13). Moreover, SG is technically easier to perform since fewer anastomoses are required. For these reasons, in 2014 it became for the first time the most widely used bariatric surgery technique worldwide, overtaking GB (10).

Intense scientific debate has arisen in recent years regarding the suitability of SG as the new gold standard in bariatric surgery and the clinical considerations to be taken into account when electing one technique or the other. Prior to the SLEEVEPASS and SM-BOSS trials, a few randomised controlled trials in bariatric surgery were available, most of them focused on type 2 diabetes and with short-term follow-up (around 1 year) $(12,14)$. In this respect, the STAMPEDE study (15) assessed 1- and 5-year outcomes in 150 patients with type 2 diabetes and body mass index of $27-43 \mathrm{~kg} / \mathrm{m}^{2}$. These patients were assigned either to intensive medical therapy alone or intensive medical therapy plus GB or SG. The study concluded that bariatric surgery was superior to conventional treatment; however, results were similar between techniques regarding type 2 diabetes remission (primary outcome) and weight loss.

\section{The SLEEVEPASS and SM-BOSS trials}

In the SLEEVEPASS study reported by Salminen et al. (1), 240 patients with a mean age of 48 years and mean body mass index of $45.9 \mathrm{~kg} / \mathrm{m}^{2}$ were randomly assigned to GB or SG; of those, 193 (80.4\%) completed the 5-year followup. On the other hand, in the SM-BOSS trial reported by Peterli et al. (2), 217 patients with a mean age of 45.5 years and mean body mass index of $43.9 \mathrm{~kg} / \mathrm{m}^{2}$ were also assigned to one of the bariatric surgery procedures (110 to GB and 107 to SG); of these, 94.5\% [205] were followed up for 5 years. Results of both trials on the mid-term efficacy of SG versus GB in terms of weight loss, comorbidities evolution, quality of life and complications are shown in the Table 1.

\section{What similarities were found between GB and SG at 5 years of follow-up in the SLEEVEPASS and SM-BOSS studies?}

Both trials established weight loss outcomes as primary objectives and found no clinically- significant differences. In the SLEEVEPASS, the 5-year percentage of excess weight loss was significantly higher with GB; however, it was considered not clinically significant since criteria of equivalence established in the design of the study were not fulfilled. Moreover, the SM-BOSS found no differences in the percentage of body mass index loss, and slight differences in the 5 -year body mass index (around $1 \mathrm{~kg} / \mathrm{m}^{2}$ ) were found between studies.

SG and GB did not differ either in terms of type 2 diabetes remission, concurring with the short-term results reported in other studies $(15,16)$. Finally, both operations were equivalent in terms of quality of life and late complications (Table 1). This contrasts with previous studies which considered the restrictive procedure "easier" compared to GB owing to a lower risk of associated complications (17).

\section{What differences were found between GB and SG at 5 years of follow-up in the SLEEVEPASS and SM-BOSS trials?}

The main discrepancy in outcomes between the studies was linked to hypertension remission, which was higher in the GB group in the SLEEVEPASS but not in the SM-BOSS. This divergence can be explained at least in part by the different criteria used to define remission in those studies (Table 1).

Furthermore, the main metabolic difference between techniques was related to lipid disorders. Both studies detected improvements in high-density lipoprotein (HDL) cholesterol and triglyceride levels with no differences between groups; however, low-density lipoproteins (LDL) cholesterol reduction was clearly greater with GB. These results concur with those of a cohort study by our group in which 5-year lipid profile evolution was compared between blood glucose (BG) and SG (18). Taking into account the different evolution of lipid fractions we considered it more appropriate to evaluate hypertriglyceridaemia, low HDL cholesterol and high LDL cholesterol remission separately than whole dyslipidaemia remission alone. We found the proportion of patients who had achieved normal LDL cholesterol levels at 5 years post-GB to be higher after GB [30/49 (61.2\%)] than SG [6/23 (26.1\%); P=0.005].

A further clear difference between procedures was the effect on gastroesophageal reflux. In this respect, GB improved gastroesophageal reflux in a high percentage of patients in the SM-BOSS study; however, it worsened more frequently with SG. Furthermore, the majority of reoperations in patients undergoing SG in the SLEEVEPASS 


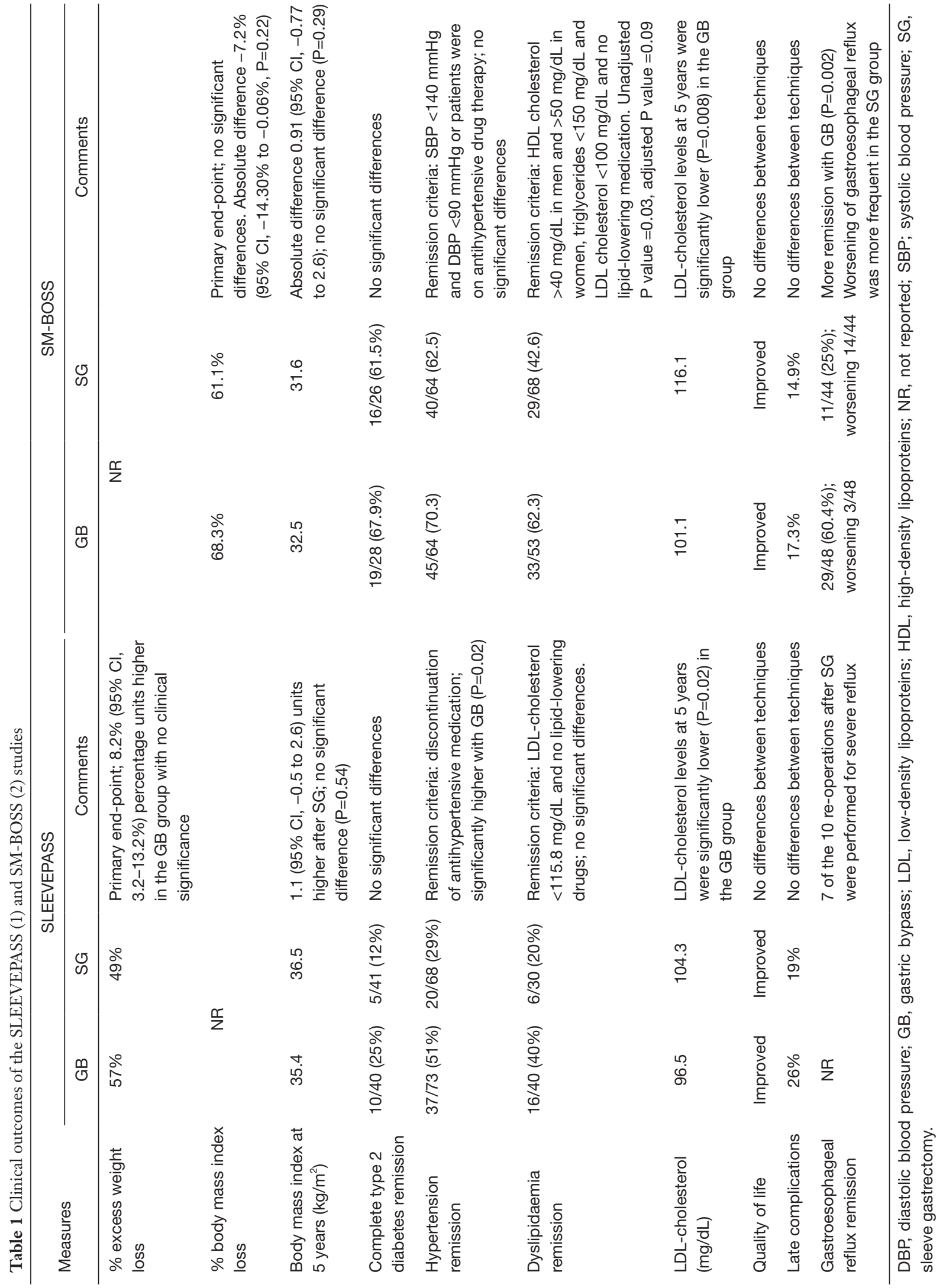


were due to gastroesophageal reflux.

\section{What are the implications in clinical practice?}

Firstly, these studies showed SG to be an effective bariatric surgery technique in the mid-term. This reinforces the dramatic rise in its use in the last decade and ensures its indication in the future. Secondly, the superiority of GB in terms of gastroesophageal reflux remission and lower LDL cholesterol should be taken into account when choosing between techniques in a candidate for bariatric surgery.

\section{What questions are awaiting an answer?}

Despite the significance of the SLEEVEPASS and SMBOSS trials, some aspects regarding the results of both procedures are still awaiting answers. Hence, future randomised controlled trials with long-term follow-up $(>5$ years) are mandatory to confirm the results observed in those trials with mid-term follow-up (3-5 years) regarding weight loss, comorbidity evolution and adverse outcomes. Moreover, divergences found in hypertension remission between studies need to be clarified with further studies focused on the mid-term effects on blood pressure. Finally, it would also be of great interest to identify factors related to comorbidity remission in the long-term after bariatric surgery. In this respect, previous publications with shortand mid-term follow-up described factors related to the remission rates of diverse obesity comorbidities $(19,20)$.

\section{Acknowledgements}

We thank Miss Christine O'Hara for review of the English version of the manuscript.

\section{Footnote}

Conflicts of interest: The authors have no conflicts of interest to declare.

\section{References}

1. Salminen P, Helmiö M, Ovaska J, et al. Effect of laparoscopic sleeve gastrectomy vs laparoscopic Roux-en-Y gastric bypass on weight loss at 5 years among patients with morbid obesity: the SLEEVEPASS randomized clinical trial. JAMA 2018;319:241-54.

2. Peterli R, Wolnerhanssen BK, Peters T, et al. Effect of laparoscopic sleeve gastrectomy vs laparoscopic Rouxen-Y gastric bypass on weight loss in patients with morbid obesity: the SM-BOSS randomized clinical trial. JAMA 2018;319:255-65.

3. NCD Risk Factor Collaboration (NCD-RisC). Worldwide trends in body-mass index, underweight, overweight, and obesity from 1975 to 2016: a pooled analysis of 2416 population-based measurement studies in 128.9 million children, adolescents, and adults. Lancet 2017;390:2627-42.

4. Finucane MM, Stevens GA, Cowan MJ, et al. National, regional, and global trends in body-mass index since 1980: systematic analysis of health examination surveys and epidemiological studies with 960 country-years and 9.1 million participants. Lancet 2011;377:557-67.

5. Benaiges D, Pedro-Botet J, Flores-Le Roux JA, et al. Past, present and future of pharmacotherapy for obesity. Clin Investig Arterioscler 2017;29:256-64.

6. Wadden TA, Neiberg RH, Wing RR, et al. Four-year weight losses in the Look AHEAD study: factors associated with long-term success. Obesity 2011;19:1987-98.

7. Sjöström L, Lindroos AK, Peltonen M, et al. Lifestyle, diabetes, and cardiovascular rsk factors 10 years after bariatric surgery. N Engl J Med 2004;351:2683-93.

8. Buchwald H, Avidor Y, Braunwald E, et al. Bariatric surgery: a systematic review and meta-analysis. JAMA 2004;292:1724-37.

9. Laferrère B, Pattou F. Weight-independent mechanisms of glucose control after Roux-en-Y gastric bypass. Front Endocrinol (Lausanne) 2018;9:530.

10. Angrisani L, Santonicola A, Iovino P, et al. Bariatric surgery and endoluminal procedures: IFSO Worldwide Survey 2014. Obes Surg 2017;27:2279-89.

11. Regan JP, Inabnet WB, Gagner M, et al. Early experience with two-stage laparoscopic Roux-en-Y gastric bypass as an alternative in the super-super obese patient. Obes Surg 2003;13:861-4.

12. Schauer PR, Kashyap SR, Wolski K, et al. Bariatric surgery versus intensive medical therapy in obese patients with diabetes. N Engl J Med 2012;366:1567-76.

13. Benaiges D, Más-Lorenzo A, Goday A, et al. Laparoscopic sleeve gastrectomy: more than a restrictive bariatric surgery procedure? World J Gastroenterol 2015;21:11804-14.

14. Mingrone G, Panunzi S, De Gaetano A, et al. Bariatric surgery versus conventional medical therapy for type 2 diabetes. N Engl J Med 2012;366:1577-85.

15. Schauer PR, Bhatt DL, Kirwan JP, et al. Bariatric surgery 
versus intensive medical therapy for diabetes - 5-year outcomes. N Engl J Med 2017;376:641-51.

16. Kehagias I, Karamanakos SN, Argentou M, et al. Randomized clinical trial of laparoscopic Roux-en-Y gastric bypass versus laparoscopic sleeve gastrectomy for the management of patients with $\mathrm{BMI}<50 \mathrm{~kg} / \mathrm{m}^{2}$. Obes Surg 2011;21:1650-6.

17. Hutter MM, Schirmer BD, Jones DB, et al. First report from the American College of Surgeons Bariatric Surgery Center Network. Ann Surg 2011;254:410-20; discussion 420-2.

18. Climent E, Benaiges D, Flores-Le Roux JA, et al.

Cite this article as: Benaiges D, Climent E, Goday A, Julià H, Flores-Le Roux JA, Pedro-Botet J. Mid-term results of laparoscopic Roux-en-Y gastric bypass and laparoscopic sleeve gastrectomy compared - results of the SLEEVEPASS and SMBOSS trials. Ann Transl Med 2018;6(Suppl 1):S83. doi: 10.21037/ atm.2018.10.70
Changes in the lipid profile 5 years after bariatric surgery: laparoscopic Roux-en-Y gastric bypass versus laparoscopic sleeve gastrectomy. Surg Obes Relat Dis 2018;14:1099-105.

19. Benaiges D, Flores-Le-Roux JA, Pedro-Botet J, et al. Impact of restrictive (sleeve gastrectomy) vs hybrid bariatric surgery (Roux-en-Y gastric bypass) on lipid profile. Obes Surg 2012;22:1268-75.

20. Flores L, Vidal J, Canivell S, et al. Hypertension remission 1 year after bariatric surgery: predictive factors. Surg Obes Relat Dis 2014;10:661-5. 